\section{Paramedics possibility to withhold resuscitation}

\section{Dear Editor}

We write in response to the letter ' $U K$ paramedics can legally withhold resuscitation in defined circumstances' by Bronnert et al. First, we have to mention that our article 'Paramedics experiences and expectations concerning advance directives: A prospective, questionnaire-based, bi-centre study' ${ }^{1}$ is correct in stating that it is not legally clear-cut whether UK paramedics may withhold resuscitation. We stated that

in most countries (e.g. Germany, United Kingdom) it is not legally clear-cut whether paramedics may withhold resuscitation in prehospital emergency medical care (regardless of the existence of an advance directive). Paramedics usually must initiate full resuscitation of patients without vital signs. ${ }^{1}$ (emphasis not in the original)

Therefore, the correct term seems to be that 'In England and Wales paramedics can legally withhold resuscitation in defined circumstances'. We also feel that it is inappropriate to compare the findings of our investigation to those of Marco et al. ${ }^{2}$ There is an important difference between a paramedic and a prehospital emergency physician. ${ }^{1,2}$ Marco's investigation is based on a prehospital paramedic system, while our investigation is based on a prehospital emergency physician system. It may not be appropriate to compare the two studies directly.

However, we think that it is very important to show that internationally, there are legal possibilities for paramedics to withhold resuscitation in terminally ill patients, as Bronnert et al. have shown in their article. In some countries, there are differences between legal permissions and medical guidelines. For example, in Germany, prehospital resuscitation is based on the recommendations by the European Resuscitation Council (ERC). ${ }^{3}$ Concerning withdrawing or withholding resuscitation, there are no legal permissions for German paramedics to operate according to the ERC guidelines. The most important problem seems to be that, certainly, a medical guideline cannot go against the legal framework in a country. Furthermore, there is a medical guideline available, but paramedics do not have legal permission to follow it. However, the Mental Capacity Act $(2005)^{4}$ should be legally reviewed concerning these important facts (medical guideline vs legal framework).

However, in some countries, and in specially defined circumstances, paramedics may have the legal possibility to withhold resuscitation. In future, there may be a chance to define international recommendations on withholding resuscitation in end-of-life patients by paramedics.

\section{References}

1. Taghavi MT, Simon A, Kappus S, et al. Paramedics experiences and expectations concerning advance directives: a prospective, questionnaire-based, bi-centre study. Palliat Med 2012; 26: 908-916.

2. Marco CA, Bessman ES and Kelen GD. Ethical issues of cardiopulmonary resuscitation: comparison of emergency physician practices from 1995-2007. Acad Emerg Med 2009; 16 : 270-273.

3. European Resuscitation Council. European Resuscitation Council Guidelines for Resuscitation 2010. Resuscitation 2010; 81(Suppl. 1): e1-e330.

4. The Mental Capacity Act 2005. London: HMSO, 2005.

Christoph HR Wiese and Mahmoud Taghavi Department of Anaesthesiology, University Hospital of Regensburg, Regensburg, Germany

Corresponding author: Christoph HR Wiese, Department of Anaesthesiology, University of Regensburg, Franz-Josef-Strauß-Allee 11, D-93053 Regensburg, Germany. Email:christoph.wiese@ukr.de 